\section{Gefangene helfen Jugendlichen nicht - wem dann?}

\section{Christine Graebsch}

\author{
Zum internationalen Stand der Evaluation von Gefängniskonfrontationsprogrammen \\ nach dem Muster von »Scared Straight»
}

$\checkmark$ efängnisbesuchsprogramme in kriminalpräventiver $\mathrm{Ab}$ sicht haben international seit einigen Jahrzehnten Konjunktur. Sie sollen Jugendliche zum Zweck der Abschreckung mit der Realität des Strafvollzugs konfrontierezn. Weithin werden solche Aversionsprogramme mit dem Namen assoziiert, unter dem eine TV-Dokumentation sie 1979 in den USA bekannt machte: »Scared Straight«. Die deutsche Ausstrahlung des Films übersetzte dies mit: »Aus Angst straffrei $\ll$.

Auch die Gefängnisbesuchsprogramme selbst sind längst in der Bundesrepublik angekommen. In der JVA Geldern gibt es das sogenannte Puschmann-Projekt; im Hamburger Strafvollzug das Projekt »Gefangene helfen Jugendlichen « und in Bremen versucht eines unter dem Namen »Knast ist nicht cool « Fuß zu fassen. Sie richten sich sämtlich an Jugendliche, die als delinquent in Erscheinung getreten sind bzw. diesbezüglich für gefährdet gehalten werden. Kern aller Programme sind Konfrontationen mit Gefangenen, bei denen diese sich mit ihrer Lebensgeschichte als Negativvorbild für die Jugendlichen präsentieren. Die Gefangenen wollen den Jugendlichen verdeutlichen, wie sich auch ihr Leben weiterentwickeln werde, wenn sie nicht noch rechtzeitig die Entscheidung zu einer Umkehr träfen. Die entsprechende Einsicht soll einen kriminalpräventiven Effekt nach sich ziehen. Ein solcher ist aber nicht nur theoretisch schwerlich begründbar, sondern auch durch Evaluationsstudien aus mehreren Ländern empirisch widerlegt. Die Gefängnisbesuchsprogramme erfreuen sich dennoch einer kontinuierlichen Beliebtheit.

\section{"Scared Straight«: Das Juvenile Awareness Project (JAP) in New Jersey}

Es war das »Juvenile Awareness Project « aus New Jersey, 1975 im dortigen Hochsicherheitsgefängnis von Langzeitgefangenen (»Lifers«) initiiert (vgl. zum JAP und seiner Evaluation Finckenauer 1982), das über das Fernsehen als »Scared Straight « bekannt wurde. Wegen seiner Berühmtheit und Vorbildfunktion für andere Programme soll es auch hier als einführendes Beispiel dienen. Um die Jugendlichen von einem an Straftaten orientierten Leben abzubringen, aber auch um ihr eigenes Bild in der Öffentlichkeit durch verantwortungsbewusstes Verhalten zu verbessern, knüpften die Gefangenen an diejenigen Kompetenzen an, die ihnen am wenigsten abgesprochen werden konnten. Als Experten ihrer eigenen Situation nahmen sie für sich in Anspruch, den Jugendlichen eine kriminalpräventiv-erzieherische Botschaft sehr viel glaubwürdiger und damit effektiver vermitteln zu können als dies den Fachleuten aus Sozialarbeit, Justiz, Schule usw. möglich ist.

Bei Ankunft im Gefängnis erhielten die ausgewählten Jugendlichen zunächst eine Einführung von einem Bediensteten, der sie darauf hinwies, dass es sich nicht um eine »Sightseeing Tour « handle und sie aufforderte, ihre Zigaretten abzugeben. In der sich anschließenden Gruppensitzung stellten die »Lifers « in derben Worten die Härte des Gefängnisalltags mit seinen völlig fremdbestimmten Abläufen, seiner unpersönlichen Atmosphäre, ungenießbarem Essen, Einsamkeit und allgegenwärtiger Gewalt bis hin zu Mord, Vergewaltigung, sexueller Versklavung sowie der Häufigkeit von Selbsttötungen dar. Anschließend folgte ein gemeinsamer Gang durch die Anstalt, bei dem eine Isolationszelle für Disziplinarmaßnahmen gezeigt wurde. Es waren auch an Gittern rüttelnde und dort zuvor eigens postierte - Gefangene zu sehen, die darum flehten, von den Qualen des Gefängnislebens erlöst zu werden. Das von Anfang an auf Lernen durch Einschüchterung ausgerichtete Programm wurde im Laufe der Zeit zunehmend in Richtung einer Schock-Konfrontation orientiert, da die »Lifers « glaubten, die Jugendlichen würden nur so hinreichend beeindruckt. Sie erniedrigten die Jugendlichen daher fast real so, wie es bei neuen Gefangenen üblich ist. Wenn dies auch auf die verbale Komponente beschränkt blieb, so ging es doch hin bis zur konkreten Androhung einer Vergewaltigung für den Fall, dass ein Jugendlicher tatsächlich einmal in diesem Gefängnis enden würde. Einen durchaus erschreckenden Eindruck von alledem vermittelt der Film »Scared Straight «, der aufgrund seiner Brutalität in Sprache und Darstellung erst nach Überwindung einiger Widerstände im US-amerikanischen Fernsehen gezeigt werden konnte. Verbreitet wurden mit ihm aber dann auch Erfolgsmeldungen, wonach 80-90\% der zuvor als notorisch delinquent bekannten Jugendlichen nach dem Gefängnisbesuch ihr Leben hin zu einem gesetzestreuen geändert hätten. Dies wäre selbst dann sehr überraschend, wenn man Abschreckungsdoktrin und Aversionstechniken, auf denen die Programme zumindest implizit beruhen, als theoretisch plausible Grundlage für kriminalpräventive Wirkungen akzeptierte. Erfolge diesen Ausmaßes sind nicht nur im Bereich Kriminalprävention mehr als ungewöhnlich, sie sollten auch noch mit einem nahezu kostenlosen Programm erreicht worden sein, und dies sogar innerhalb eines nur einzigen Tages.

\section{"Gefangene helfen Jugend- lichen« und andere deutsche Gefängnisbesuchsprogramme}

In Deutschland gibt es zwei bekanntere Gefängnisbesuchsprogramme und eine unbekannte Zahl weiterer. Das sogenannte Puschmann-Projekt in der JVA Geldern (vgl. dazu die Darstellung in ZfStrVo 2000, S. 367) begann im Jahre 1991 und dürfte damit das erste Gefängniskonfrontationsprogramm in Deutschland gewesen sein. Es entwickelte sich aus dem Gefangenengesprächskreis des ehrenamtlichen Vollzugshelfers Johannes Puschmann. Das Projekt verfolgt vorrangig das Ziel der Kriminalprävention, dieses soll nicht allein aber offensichtlich unter anderem über Abschreckung erreicht werden. Im Vordergrund soll eine echte und persönliche Begegnung zwischen Langzeitgefangenen und Jugendlichen stehen. Ein Besuch im Strafvollzug dauert dabei in der Regel fünf bis sechs Stunden und läuft nach den Vorgaben eines Konzeptpapiers von Puschmann ab. Danach werden die Jugendlichen zunächst vor der Anstaltspforte begrüßt. Dann werden ihre Personalien überprüft und es finden Kontrollen der Art statt, wie sie für einen Besuch in der JVA üblich sind. Zwei der Jugendlichen gehen dann zur Kleiderkammer und werden mit Anstaltskleidung ausgestattet, die sie bis zum Ende der Veranstaltung tragen. Anschließend wird jeder dieser Jugendlichen alleine für 10 bis 15 Minuten in eine Zelle eingesperrt. Dabei soll er sich Gedanken darüber machen, ob er dort "für längere Zeit untergebracht werden möchte «. Die anderen Jugendlichen treffen mit den Gefangenen im Besucherraum der Anstalt zusammen. Alle, zusätzlich auch Sozialarbeiter, sitzen um einen großen runden Tisch, wobei jeder Jugendliche zwischen zwei Gefangenen Platz nehmen muss. So sollen für das Programm kontraproduktive Interaktionen zwischen den Jugendlichen verhindert werden. Das Gespräch wird von Gefangenen geführt, die zunächst den Alltag im Knast allgemein erläutern. Dann besichtigen alle Jugendlichen zusammen mit 
3 Gefangenen die Zelle, in der jugendliche Neuzugänge untergebracht werden. Die Gefangenen stellen dort Fragen, die die Jugendlichen dazu zwingen sollen sich vorzustellen, dort selbst eingesperrt zu sein. Anschließend findet, wieder im Besucherraum, ein Erfahrungsaustausch mit den beiden Jugendlichen statt, die umgekleidet und in eine Zelle gesperrt worden waren. Sie müssen schildern, wie sie sich fühlten, zum Beispiel als sie durch Abgeben ihrer Kleidung vom Individuum zu einer »Nummer" degradiert wurden. Es folgt ein Diavortrag über das Leben hinter Gittern und ein gemeinsames Mittagessen mit der Gelegenheit zu Einzelgesprächen, die durch eine »gemischte " Sitzreihenfolge angeregt werden sollen. Dabei offenbaren die Gefangenen ihre Lebensgeschichten inklusive Straftaten und die Jugendlichen sollen ihre Lebenssituation darstellen. Gemeinsam soll dann nach Parallelen im Lebensverlauf gesucht werden, um weiterer Straffälligkeit der Jugendlichen entgegenzuwirken. Offenbar werden viermal jährlich Jugendliche von der Jugendgerichtshilfe aus Düsseldorf und Wesel in die JVA Geldern geschickt (Bock 2000). Wesentlicher Bestandteil des Programms ist die Nachbereitung des Gefängnisbesuchs in sozialen Trainingskursen der Jugendgerichtshilfe. Die Aufarbeitung der Denk- und Handlungsanstöße, die die Jugendlichen im Gefängnis erfahren haben, gilt als wichtigste Voraussetzung für den Erfolg eines Gefängnisbesuchs.

Umfangreiche Öffentlichkeitsarbeit wird von dem Projekt »Gefangene helfen Jugendlichen « aus Hamburg betrieben (vgl. vor allem deren Homepage unter http://www.gefangenehelfen-jugendlichen.de). Es läuft seit 1999 in der Anstalt II am Hasenberge in Hamburg-Fuhlsbüttel. Die Behörde für Schule, Jugend und Berufsbildung, zusammen mit der Justizbehörde für die Projektkoordination zuständig, wählt in Zusammenarbeit mit Schulen und Jugendeinrichtungen Jungen aus, die für den freiwilligen Besuch im Männervollzug in Frage kommen. GhJ richtet sich an Jugendliche zwischen 13 und 21 Jahren. Es soll weniger der Abschreckung dienen, als eine Chance für die Jugendlichen sein, mit Strafgefangenen als Experten ihrer eigenen Situation ins Gespräch zu kommen. Für die Gefangenen bedeutet dieses Engagement gesellschaftliche Verantwortung und Verantwortung für ihre Taten zu übernehmen. Das Projekt ist auf Initiative von Gefangenen entstanden, und es hat das Ziel, in Hinblick auf Straftaten gefährdete Jugendliche durch Konfrontation von einer kriminellen Karriere abzubringen, sie so vor dem Gefängnis sowie Jugendliche ohne deutschen Pass vor der Abschiebung zu bewahren. Bei ihrem Besuch in "Santa Fu « sehen die Jungen zunächst einen Film über den Alltag im Gefängnis, und sie werden dann einige Minuten lang in eine Zelle gesperrt, um das Gefühl des Eingeschlossenseins kennen zu lernen. Die Jugendlichen, die zunächst in Gruppen in das Gefängnis geführt werden, unterhalten sich danach einzeln mit Gefangenen. Diese Gespräche, bei denen die Gefängnisinsassen ihr Leben vor und nach der Inhaftierung erzählen, stellen den Kern des Programms dar, an den sich auch die Hoffnung auf eine präventive Wirkung vorrangig knüpft. Ein Besuch dauert drei Stunden, und die Jugendlichen dürfen jeweils nur einmal kommen. Die Botschaft, die die Gefangenen den Jugendlichen dabei vermitteln wollen, lässt sich am besten mit dem Titel einer Ausstellung zusammenfassen, die ebenfalls dazu dienen sollte, einem über die Medien verbreiteten Bild des Gefängnisalltags entgegenzuwirken. Diese Ausstellung, bei der künstlerische Werke von Gefangenen in einem Hamburger Gerichtsgebäude präsentiert wurden, und bei der auch das geplante Gefängnisbesuchsprogramm vorgestellt wurde, hieß »Knast ist nicht cool .

\section{»Knast ist nicht cool« in Bremen}

Gerade so ist auch ein mit dem GhJ aus Hamburg kooperierendes Projekt benannt, das sich gerade in der JVA Bremen etabliert hat. Jedenfalls Presseberichten zufolge scheint es eher konfrontativer angelegt sein als das GhJ (vgl. etwa Süddeutsche Zeitung vom 23.7.2005). Nach dem JVA-Besuch und Gesprächen mit Gefangenen sollen die Jugendlichen eine Art Fragebogen ausfüllen. In diesem müssen sich die Jugendlichen zwischen ihnen in den Mund gelegten Antwortvorgaben entscheiden (Polaritätenbaum), wovon die einen für ein Leben mit, die anderen für eines ohne Straftaten stehen. In diesem Vorgehen wird auch eine Erfolgskontrolle für das Projekt gesehen. Aber obwohl von einem Psychologen initiiert, ist es den anderen, von Gefangenen konzipierten Programmen in seiner theoretischen Fundierung keineswegs überlegen.

\section{Evaluation des JAP}

Regelmäßig finden sich in Medienberichten über Gefängnisbesuchsprogramme zumindest große Erwartungen an deren kriminalpräventive Wirkung, oft aber auch schon die Behauptung, diese würden durch die Programme erreicht. Erfolgsmeldungen über das JAP, die sich auch in Zeitungsberichten fanden, stützten sich auf Briefe von Mitarbeitern derjenigen Behörden, die Jugendliche für eine Programmteilnahme ausgewählt hatten sowie auf Antworten von Erziehungsberechtigten auf einen Fragebogen der »Lifers«-Gruppe, in denen nach bemerkbaren Veränderungen im Verhalten der Jugendlichen gefragt worden war.

\section{Zweifel an der Wirksamkeit}

An der kriminalpräventiven Wirkung des JAP kamen allerdings nach und nach Zweifel auf. Sie gründeten sich nicht zuletzt darauf, dass schon bald über 14. 000 zuvor als delinquent auffällige Jugendliche einen Besuch im Gefängnis absolviert hatten, die registrierte Jugendkriminalität sich in New Jersey aber nicht veränderte (Cook/Spirrison 1992, S. 91). Nachdem erhebliche Widerstände seitens der Programmbefürworter überwunden worden waren, konnte schließlich die externe Evaluation Finckenauers beginnen. Entsprechend dem Forschungsplan sollte die Studie ein experimentelles Design mit randomisierter Zuweisung der Jugendlichen $\mathrm{zu}$ einer Experimentalgruppe (Besuch des JAP) bzw. einer Kontrollgruppe (ohne solche Maßnahme) haben. Die beteiligten Behörden verweigerten aber bei der Zufallszuweisung vielfach ihre Kooperation, und es passierten dabei diverse Fehler wohl nicht nur unabsichtlich. Dadurch konnte der entscheidende methodische Vorteil, den man sich von Zufallsexperimenten erhofft, nämlich eine weitgehende Vergleichbarkeit der Gruppen mit und ohne Programmteilnahme herzustellen, nicht realisiert werden. Allerdings analysierte Finckenauer seine Daten anschließend im Hinblick auf eine Vielzahl solcher Faktoren, die bekanntermaßen zu Unterschieden in der Programmwirkung führen können (Alter, Geschlecht, vorherige strafrechtliche Auffälligkeit usw.) und fand dabei keinerlei systematische Verzerrungen. Im Ergebnis zeigte sich, dass $41 \%$ der Jugendlichen aus der Experimental-, aber nur 11\% derjenigen aus der Kontrollgruppe nach 6 Monaten strafrechtlich in Erscheinung getreten waren. Zudem hatte die Experimentalgruppe schwerwiegendere Delikte begangen. Nicht nur die utopischen Erfolgsmeldungen blieben also unbestätigt, das Programm erwies sich demnach sogar als in kriminalpräventiver Hinsicht schädlich ${ }^{1}$.

\section{Wirkung der Evaluation des JAP}

Nachdem die von Finckenauer 1982 in einem Buch veröffentlichten Ergebnisse der Evaluation bekannt waren, wurde das JAP aber keineswegs abgeschafft (Lundman 2001, S. 188 f.; Finckenauer / Gavin 1999, S. 124). Das Medienecho zu »Scared Straight « ist bis heute vorwiegend positiv. Im Jahre 1999, 20 Jahre nach der Ausstrahlung der ersten TV-Dokumentation zu "Scared Straight «, zeigte das US-Fernsehen dazu einen neuen Film. Dieser beschäftigte sich mit der seitherigen Entwicklung der seinerzeit gefilmten Jungen und Mädchen und verkündete erneut die Botschaft kriminalpräventiven Erfolgs in über 80\% der Fälle. Die Ergebnisse der Studie von Finckenauer müssten dann eben falsch sein, konstatierte der Produzent beider Filme, Shapiro (Muhammad 1999). In einem Zeitungsartikel der New York Times vom 16. Mai 1996 wurde das Programm als auf dem Höhepunkt seiner Popularität beschrieben. Wöchentlich fanden zu diesem Zeitpunkt Besuche von etwa zehn Gruppen statt, jährlich würden über 12.500 Jugendliche in das Programm einbezogen. Die Ausrichtung des Programms wurde so dargestellt, dass es sich mittlerweile vor allem an Jugendliche richte, die in besonders großen Schwierigkeiten seien, weil es auf diese am besten einwirken könne. Die Studie von Finckenauer war allerdings zu exakt dem umgekehrten Ergebnis gekommen: Es zeigte sich 
nämlich, dass das Programm stattdessen auf die zuvor nicht als delinquent aufgefallenen Jugendlichen eine etwas weniger negative Wirkung hatte (Finckenauer / Gavin 1999, S. 124, 87 f.). Die Ergebnisse von Finckenauer wurden also offenbar auch für die inhaltliche Weiterentwicklung des Projekts in keiner Weise berücksichtigt. In dem Artikel der New York Times wird nunmehr eine Erfolgsrate von $51 \%$ behauptet, ohne dafür eine Quelle anzugeben. Außerdem sollen demnach die »Lifers « in New Jersey den Jugendlichen inzwischen Informationen und Statistiken über das Gefängnis geben, statt Horrorgeschichten darüber $\mathrm{zu}$ erzählen. In demselben Artikel wird allerdings ein Szenario beschrieben, wonach die Jugendlichen eben doch anhand von Fotos und Geschichten (erfolgreich) negativ zu beeindrucken versucht worden seien. Demnach dürfte sich an den Konfrontationen als Kern des Programms nichts geändert haben.

Auch bemüht man sich durchaus weiterhin, die Effektivität des "Juvenile Awareness Projects « nachzuweisen. Dies geschieht offenbar mit einer ganzen Reihe unterschiedlicher Herangehensweisen, überwiegend aber über eine Korrespondenz mit den Jugendlichen, die das Gefängnis besucht haben und den »Lifers« später über ihre Erfolge berichten - also exakt mit der schon vor der wissenschaftlichen Evaluation angewendeten »Methode«, an deren Unzulänglichkeit sich seither nichts geändert hat (Finckenauer/Gavin 1999, S. 126).

\section{Experimentelle und quasi-expe- rimentelle Evaluation diverser Gefängnisbesuchsprogramme}

Ein hinsichtlich der kriminalpräventiven Wirkung negatives Ergebnis brachte aber nicht nur die Evaluation des berühmten JAP. Petrosino et al. (2000, 2003) stellten die Ergebnisse dieser sowie acht weiterer Studien zusammen, die alle ein echt experimentelles Forschungsdesign (mit Randomisierung oder Quasi-Randomisierung, d. h. Alternierungsverfahren) hatten:

Bereits im Jahre 1967 evaluierte das Michigan Department of Corrections die Wirkung von Besuchsgän- gen Jugendlicher durch das Gefängnis, wenn auch hier wohl noch kein Element der Konfrontation mit Gefangenen enthalten war. Der Forschungsbericht ist unveröffentlicht, und über die Studie ist wenig bekannt. Das ist gerade angesichts der Tatsache erstaunlich, dass bereits diese frühe Evaluation den späteren vollmundigen Erfolgsversprechungen der Gefängnisbesuchsprogramme diametral entgegen stand. Von den 28 männlichen Jugendlichen der Experimentalgruppe waren nämlich $43 \%$ nach 6 Monaten erneut strafrechtlich in Erscheinung getreten, wohingegen dies bei den 30 Jungen der Kontrollgruppe lediglich $17 \%$ waren. Die Forscher zogen den Schluss, dass die kriminalpräventive Wirkung nicht bestätigt werden konnte und mit einer Wahrscheinlichkeit von neun Zehnteln davon ausgegangen werde könne, dass das Projekt sogar einen Bumerang-Effekt bewirke, sprich: die Begehung von Straftaten, erst hervorrufe.

1979 kam die Greater Egypt Regional Planning \& Development Commission mit ihrer Evaluation der $\gg$ Menard Prison Tours « zu einem weniger drastischen, aber in die gleiche Richtung weisenden Ergebnis. Dieses Programm war als direkte Konsequenz der Erfolgsmeldungen zu »Scared Straight « entstanden, sollte in den Konfrontationen aber weniger provokativ sein. Während aus der Experimentalgruppe $17 \%$ der Jungen in der bis zu 15-monatigen Follow-upPeriode erneuten oder auch erstmaligen Kontakt mit der Polizei hatten, waren es aus der Kontrollgruppe nur $12 \%$. Das schlechtere Abschneiden der Experimentalgruppe war nicht signifikant, das Programm erwies sich damit aber immerhin als in kriminalpräventiver Hinsicht wirkungslos. Demgegenüber hatten Eltern und Lehrer die Gefängnisbesuche einmütig unterstützt und waren damit ebenso zufrieden wie die Jugendlichen selbst. Auch die an der Konfrontation beteiligten Gefangenen äußerten sich weithin positiv. Die Forscher kommen zu der Einschätzung, dass immerhin für diese das Programm wohl ein Erfolg gewesen sei.

Ebenfalls aus dem Jahr 1979 stammen die Ergebnisse der Evaluation des wiederum in Michigan durchgeführten JOLT (»Juvenile Offenders Learn Truth«). Allerdings erschien nur ein Teil der für eine freiwillige Teilnahme ausgewählten Jungen tatsächlich zu einem Gefängnisbesuch, woraufhin die Forscher auch die Kontrollgruppe in einer Weise verkleinerten, die systematische Verzerrungen nicht ausschließen kann (Homant/Osowski 1981). Wenn sich insofern auch Zweifel an den Ergebnissen nicht ausräumen lassen, so wurden nach diesen doch wiederum die kriminalpräventiven Hoffnungen jedenfalls nicht bestätigt, da sich keine signifikanten Unterschiede zwischen den Gruppen fanden.

Die experimentelle Evaluation des »Insiders Program « in Virginia von 1981 ist die einzige mit einem Ergebnis in die Richtung, die bei einem erfolgreichen Präventionsprogramm zu erwarten wäre, indem nämlich die Experimentalgruppe langfristig geringere Rückfallraten zu verzeichnen hatte als die Kontrollgruppe. Allerdings zeigten sich diese Resultate noch nicht nach 6 , sondern erst nach 9 und 12 Monaten, als nur noch rund die Hälfte des ursprünglichen Samples erreicht werden konnte. Die dann noch von der Studie erreichten Jugendlichen der Experimentalgruppe könnten eine Untergruppe gewesen sein, die mit Straftaten unabhängig von der Programmwirkung seltener in Erscheinung traten. Petrosino et al. (2003, S. 16) sprechen zwar von Analysen, die dies angeblich ausschließen sollen. Sie benennen diese aber nicht näher, und der Forschungsbericht der Evaluation ist unveröffentlicht. Eine Bestätigung kriminalpräventiven Erfolgs muss letztlich auch hier zweifelhaft bleiben. Schließlich spricht wenig dafür, dass Effekte eines nur eintägigen Erlebnisses erst nach so langer Zeit eintreten, und Hinweise auf eine solche zeitverzögerte Wirkung finden sich sonst nirgendwo.

Bei der experimentellen Evaluation des Programms »Face to Face« in Texas wurde die Wirkung eintägiger Aufenthalte im Gefängnis erforscht. Die unter Bewährung stehenden Jungen sollten dabei kurzzeitig genau so leben wie Gefangene. In einem faktoriellen Design wurden zusätzliche Elemente verglichen; so gab es Experimental- und Kontrollgruppen jeweils mit einer und ohne eine anschließende Gruppenberatung durch einen Bewährungshelfer bzw. mit/ohne Erziehungsberatung für die Eltern. Anhand der in den Gerichtsstatistiken registrierten Rückfallraten schnitt die ohne all dies gebliebene Kontrollgruppe besser ab (28\%) als die Treatmentgruppen, gleichgültig ob diese einen Besuch absolvierten, Beratung erhielten oder beides (36\% - 39\%) - wenn auch die Unterschiede zwischen beiden Gruppen nicht signifikant waren. Neben der registrierten wurde in dieser Studie auch die selbstberichtete Kriminalität berücksichtigt, wobei das Ergebnis in die umgekehrte Richtung ging (59\% bei den Experimentalgruppen, $69 \%$ in der Kontrollgruppe), aber gleichfalls der Unterschied nicht signifikant war. Hier wird ein Problem experimenteller Evaluationsstudien deutlich, die auf den Vorteil randomisierter Gruppenzuweisung und Vergleichbarkeit der Gruppen setzen, aber ohne theoretische Fundierung auszukommen meinen. Während es schon bei den vorangegangenen negativen Ergebnissen zur kriminalpräventiven Wirkung der Programme an einer Erklärung für diese fehlte, wird dieses Problem durch Hinzuziehung der Daten zu selbstberichteter Delinquenz keineswegs gelöst, es tritt vielmehr erst verstärkt zu Tage. So sah sich der Forscher (Vreeland 1981 unveröffentlicht, wiedergegeben bei Petrosino et al. 2000, S. 364) nicht in der Lage, die Divergenzen zwischen den Messmethoden zu erklären, die doch beide als Indikator für kriminalpräventiven Erfolg gängig sind. Trotz allem lässt sich aber festhalten, dass eine kriminalpräventive Wirkung von Gefängnisbesuchsprogrammen mit Konfrontationselement auch mit dieser Studie nicht nachgewiesen werden konnte.

Lewis (1981) evaluierte das neben dem JAP ebenfalls recht bekannt gewordene und wohl älteste Programm »SQUIRES« (»San Quentin Utilization of Inmate Resources, Experience and Studies «). In dem kalifornischen Hochsicherheitsgefängnis wurden die Gefängnisbesuche an drei aufeinanderfolgenden Samstagen wiederholt. Es gab auch die ansonsten übliche Gruppensitzung, die als didaktische Konfrontation verstanden wurde und bei der jeder Junge einem Gefangenen individuell zugeordnet war. Auf einem »heißen Stuhl « sitzend, sollten die Jungen zudem über von ihnen begangene Straftaten und Hintergründe berich- 
ten. Die Jugendlichen beurteilten das Programm anschließend sehr positiv und ihre Einstellungen zu Kriminalität und Polizei sollen sich in der intendierten Richtung geändert haben, was eine Voraussetzung für Verhaltensänderungen im Sinne der Programmintention ist (Lewis 1981, S. 25-44; differenzierter und, in Hinblick auf das JAP, im Ergebnis auch schon eine Wirkung auf die Einstellung von Jugendlichen ablehnend Finckenauer/Gavin 1999, S. 107-116). Dennoch schnitt auch bei diesem Experiment hinsichtlich der verhaltensbezogenen Rückfallindikatoren zumeist die Kontrollgruppe besser $a b$, die allerdings keine NonInterventions-Gruppe war, sondern sich, wie auch die Experimentalgruppe, in einer Maßnahme befand, z. B. einem Campaufenthalt. Nach 12 Monaten waren $81 \%$ der Experimentalprobanden, aber nur $67 \%$ aus der Kontrollgruppe erneut von der Polizei festgenommen worden, wobei der Unterschied zwischen den Gruppen aber nicht signifikant ist. Der Zeitraum bis zu der ersten Festnahme war allerdings bei der Experimentalgruppe signifikant länger: 4,1 Monate im Vergleich zu 3,3. Dieses Ergebnis kann nicht auf einen in der durchschnittlich etwas älteren Experimentalgruppe stärker fortgeschrittenen Maturationsprozess zurückgeführt werden (so aber Finckenauer/ Gavin 1999, S. 131), wie schon die Analyse von Lewis (1983, S. 225) zeigt. Schon weil die Jungen jedoch nach dieser längeren Zeit mit schwereren Taten in Erscheinung traten, kann auch nicht von einer positiven Teilwirkung im Sinne der Programminitiatoren und im Namen der Kriminalprävention gesprochen werden.

Mit dem »Juvenile Education Program « in Kansas wurde von Locke et al. (1986) ein Programm evaluiert, das als gegenüber dem JAP in New Jersey ausdrücklich sehr viel weniger konfrontatives angelegt war. Eine Besonderheit war auch, dass versucht wurde, Jugendliche mit Gefangenen zusammenzubringen, deren individueller Lebensstil sich ähnelte. Als abhängige Variable wurde nicht nur die registrierte, sondern auch die selbstberichtete Delinquenz der Jungen herangezogen. Es ergaben sich dabei keinerlei signifikante Unterschiede zwischen Experimental- und Kontrollgruppe. Die Entwicklung un- terhalb des Signifikanzniveaus wurde von den Forschern nicht mitgeteilt, und es gab Probleme hinsichtlich der Vollständigkeit der zuvor ausgewählten Gruppen. Im Ergebnis konnte auch mit dieser Studie kein kriminalpräventiver Effekt nachgewiesen werden.

Auch mit dem »Project Aware« in Mississippi ist von Cook/Spirrison (1992) ein Programm evaluiert worden, in dem statt auf Konfrontation auf Erziehung gesetzt wurde. Die anhand von durchnummerierten Jugendgerichtsakten alternierend dem Programm und der Kontrollgruppe zugewiesenen Jungen schnitten hinsichtlich der Häufigkeit und Schwere von nach 12 und 24 Monaten registrierten Delikten nicht signifikant unterschiedlich ab, die Kontrollgruppe aber bei insgesamt sinkender Auffälligkeit beider Gruppen zumeist geringfügig besser. Zusätzlich wurde der Erfolg des Programms auch an der Entwicklung des unentschuldigten Fehlens in der Schule gemessen. Während sich hinsichtlich der Fehltage keinerlei Unterschiede zwischen den Gruppen feststellen ließen, wurden die Jugendlichen aus der Experimentalgruppe signifikant seltener zu Schulabbrechern. Mangels theoretischer Fundierung auch dieser Studie muss allerdings völlig offen bleiben, worauf dieser Effekt zurückzuführen sein könnte. Angesichts der anderen Ergebnisse, gerade auch dieser Studie selbst, kann von einem diesbezüglich isolierten Abschreckungseffekt nicht ausgegangen werden. Selbst wenn man also in der Verhinderung von Schulabbrüchen einen indirekten positiven Erfolg bei der Prävention von (registrierter) Kriminalität sehen wollte, könnte damit eine positive Wirkung der Programme nicht belegt werden, zumal es auch an einer Erklärung fehlte, weshalb sich nicht auch Auswirkungen auf das Schulschwänzen zeigten.

So ergibt sich insgesamt anhand der experimentellen Studien sehr eindeutig, dass eine kriminalpräventive Wirkung der Gefängniskonfrontationsprogramme nicht nachweisbar ist, vielmehr einiges für die Annahme entgegengesetzter Effekte spricht. Diese Einschätzung war prinzipiell auch schon vor der "Systematic Review« von Petrosino et al. bekannt (vgl. etwa Lundman 2001 und schon 1993), die aber zusätzlich noch un-

Bei der Evaluation einzelner Programme, etwa des australischen Programms »Stay Straight « aus Victoria zeigte sich, dass es auch andere als kriminalpräventive Intentionen sein können, die zur Bestandskraft der Programme beitragen. Dort waren negative Ergebnisse bezüglich der Abschreckungswirkung früherer australischer Gefängnisbesuchsprogramme bereits bekannt, als sich ein Gefangener mit der falschen Behauptung, eines habe davon habe eine $70 \%$ ige Erfolgsrate aufgewiesen und mit dem Anliegen, ein solches Programm neu zu etablieren, an die Verantwortlichen wendete. Nachdem diese zunächst unter Hinweis auf frühere Misserfolge mit und Kontroversen über solche Programme ablehnten, gab der »Direktor General of Corrections « schließlich wider besseren Wissens nach, offenbar weil es dem Gefangenen gelungen war, 1.500 befürwortende Briefe von Personen des öffentlichen Lebens vorzulegen. Begründet wurde diese Kehrtwende mit der erwarteten Wirkung nicht des Besuchs selbst, sondern seiner Nachbereitung, deren halbherzige Umsetzung aber dafür spricht, dass es sich dabei nur um eine vorgeschobene Rechtfertigung handelte. Zudem beklagten sich Jugendliche später darüber, von Strafvollzugsbediensteten misshandelt worden zu sein, bei anderen gab es Hinweise darauf, dass sie durch den Besuch so erfolgreich verängstigt wurden, dass eine Traumatisierung zurückblieb. Geplant war die Programmteilnahme als Voraussetzung für Entlassung aus der Untersuchungshaft, praktisch wurde sie aber zu einer Netzerweiterung strafrechtlicher Sanktionen, indem sie, wohl auch gerade aufgrund ihrer Härte, von den Gerichten zunehmend als Strafmaßnahme angeordnet wurde. Die Anschuldigungen wegen Tätlichkeiten führten schließlich dazu, dass das Programm gestoppt werden konnte.

veröffentlichte Studien mit einbezog. Bereits der berühmt gewordene Bericht des Maryland-Teams Sherman et al. (1997, Neuauflage 2002, S. 338, 341, 350) über den Forschungsstand zu kriminalpräventiven Programmen verbuchte die Gefängnisbesuchsprogramme unter der Kategorie »what doesn't work «, wofür dem Team bereits eine kleiner Teil der hier angeführten Studien genügte.

Herangezogen wurde dabei insbesondere die quasi-experimentelle Evaluation des Programms »Stay Straight « auf Hawaii (Buckner/Chesney-Lind 1983), das dem JAP aus New Jersey nachgebildet war. In Abgrenzung zum JAP sollten allerdings keine Einschüchterungstechniken zur Anwendung kommen, vielmehr erzählten die Gefangenen Ereignisse aus ihrem eigenen Leben, die zur Inhaftierung geführt hatten, und sie gaben manchmal den Jugendlichen Ratschläge. Die wesentliche Botschaft bestand auch hier darin, dass sich Kriminalität nicht auszahle, und die Gefangenen dies besser als jeder an- dere wissen müssten. Während sich die experimentell angelegten Studien mit Ausnahme der von Finckenauer lediglich auf männliche Jugendliche bezogen hatten, waren hier ein Drittel der teilnehmenden Jugendlichen Mädchen. Auf der Basis von Unterlagen der Gefangenen über frühere Gefängnisbesuche einerseits und über diese Jugendlichen betreffende Polizeiakten andererseits wurden mit einem Matchingverfahren zwei Gruppen gebildet. Diese konnten dann bezogen auf die Häufigkeit erneuter Festnahmen in dem Jahr nach Programmteilnahme miteinander verglichen werden. Hinsichtlich der Anzahl von Wiederverhaftungen insgesamt ergaben sich keine signifikanten Unterschiede zwischen der Gruppe mit und der ohne Teilnahme. Eine unterhalb des Signifikanzniveaus in Hinblick auf Kriminalprävention kontraproduktive Tendenz zeigte sich nur für die Jungen der Experimentalgruppe. Diese wiesen auch signifikant häufiger Folgeverhaftungen mit sich anschließendem Gerichtsverfahren auf. Das Ergebnis bei 
den Mädchen tendierte dagegen unterhalb des Signifikanzniveaus in die erwünschte Richtung. Für die festgestellten Programmeffekte und deren Unterschiedlichkeit kann keine polizeiliche Wahrnehmung in Abhängigkeit von der Teilnahme verantwortlich gemacht werden, da die Polizei nicht darüber informiert war, wer das Gefängnis besucht hatte und wer nicht. Trotz Kontrolle vielfältiger Variablen von möglichem Einfluss kann nach den Prämissen experimenteller Forschung bei einem quasi-experimentellen Design ohne Zufallszuweisung aber nicht ganz ausgeschlossen werden, dass sich die Gruppe der zuvor von irgendwelchen Erwachsenen für eine Programmteilnahme vorgeschlagenen Jugendlichen in einer systematischen, wenn auch unbekannten, Weise von der Kontrollgruppe unterschieden. Zumindest die diversen, auch experimentellen, Studien mit negativem Ergebnis stehen einer solchen Annahme aber entgegen.

Wie gesichert das negative Resümee zu »Scared Straight « seit längerem schon ist, zeigt schließlich die Meta-Analyse von Lipsey (1992, S. 123 f.), auf die auch das MarylandTeam Bezug nahm. Unter den von Lipsey einbezogenen über 400 experimentellen und quasi-experimentellen Studien über Maßnahmen gegen Jugenddelinquenz finden sich durchaus auch evaluierte Programme mit einem in Hinblick auf Kriminalprävention positiven Effekt. Mit einer Effektgröße bei praktisch null liegen am untersten Ende der Skala Abschreckungsprogramme, wobei »Scared Straight « als Beispiel für einen Programmtyp steht, dem sogar negative Wirkungen zugesprochen werden.

Die "Systematic Review « von Petrosino et al. mündete schließlich ebenfalls in eine Meta-Analyse der experimentellen Studien $\mathrm{zu} »$ Scared Straight «. Sie konnte wegen ansonsten fehlender Daten nur auf Ergebnisse hinsichtlich offizieller Registrierung und nur auf einen 6-Monatszeitraum nach der Intervention bezogen werden. Dabei ergab sich sogar ein signifikant negativer Effekt der Programme. An diesem Ergebnis änderte sich auch dann nichts, wenn die Studie von Finckenauer aufgrund der Randomisierungsprobleme und wenn die Studie von Yarbo- rough aufgrund fehlender »no-shows« herausgenommen wurde (Petrosino et al. 2003, S. 20-25). Dieses Resultat führt bei Petrosino et al. sowie in einem »Campbell Collaboration Research Review Policy Brief « zu der zusammenfassenden Empfehlung an Politiker, ein entsprechendes Programm rigoros, d.h. experimentell, zu evaluieren, um sicherzustellen, dass sie damit nicht mehr Schaden als Nutzen hervorriefen. Eine angesichts der Ergebnisse erstaunlich zurückhaltende Empfehlung, von der man vermuten kann, dass sie von den AutorInnen der »Systematic Review « auch schon ohne deren aufwendige Durchführung gegeben worden wäre.

\section{Qualitative Studie in Norwegen}

Initiiert von einem norwegischen Strafvollzugsbediensteten, der zuvor dem JAP in New Jersey einen Besuch abgestattet hatte, wurde 1992 im norwegischen Hochsicherheitsgefängnis Ullersmo ein Programm etabliert, das dem JAP nachgebildet war, nach der Selbsteinschätzung der Verantwortlichen aber weniger konfrontativ. Mit einer abgespeckten Programmversion begaben sich die Gefangenen auch in Schulen außerhalb des Strafvollzugs. Das Gefängniskonfrontationsprogramm wurde mit einer qualitativen Studie, bestehend aus teilnehmender Beobachtung und Interviews mit den Beteiligten, von Hovland/Storvoll (in Finckenauer / Gavin 1999, Kap. 8-11) evaluiert. Diese weisen darauf hin, dass die Programme sämtlich auf dem Glauben an eine Psychologie des großen Wendepunkts beruhten. Danach werde davon ausgegangen, dass das Erlebnis nur angsteinflößend, realistisch oder gutwillig beratend genug sein müsse, um als Reaktion eine grundlegende Veränderung im Leben der Jugendlichen zu bewirken. Anders als bei den (quasi-)experimentellen Studien, die sich lediglich dafür interessierten, ob eine kriminalpräventive Wirkung messbar ist, nicht aber, wie sie begründet sein oder was ihr entgegenstehen könnte, gibt die qualitative Studie einen tiefergehenden Einblick in die relevanten Wirkungsmechanismen.

Die Jugendlichen wurden schon vor dem Besuch in Ullersmo bei einem diesem vorangehenden Treffen in der Institution beobachtet, die die meisten Personen in das Programm schickte. Dabei bestand ein Klima der Angst sowie eine Art Wettbewerb, wer sich vor den Gefangenen am meisten fürchtete, geschürt von denjenigen, die bereits zuvor an einem Besuch teilgenommen hatten. $\mathrm{Zu}$ Beginn des Aufenthalts in Ullersmo informierten die Mitarbeiter der entsendenden Institution die verantwortlichen Gefangenen kurz über die Jugendlichen und ihre Straftaten, während letztere eine Einführung durch Strafvollzugsbedienstete erhielten. Aufgrund des Vorgesprächs, von dem die Jugendlichen nichts erfahren sollten, konnten diese später von den Gefangenen individuell unterschiedlich angesprochen werden. Die in der Vergangenheit unauffälligen Jugendlichen wurden infolgedessen in Ruhe gelassen, hingegen kreisten die Gefangenen die anderen Jugendlichen ein und bliesen ihnen dabei Rauch ins Gesicht. Bei dem Zusammentreffen im Speisesaal forderten die Gefangenen von den Jugendlichen volle Aufmerksamkeit. Sie schrieen oder knallten mit Tischen, wenn sie für einen kurzen Augenblick nicht beachtet wurden, »wischten « einen Aschenbecher vom Tisch, und sie warfen Salz- und Pfefferstreuer gegen die Wände. Als ein Junge versuchte, seine Unsicherheit zu überspielen, schlug ein Gefangener mit der Faust auf den Tisch und forderte ihn auf, das Grinsen vom Gesicht verschwinden zu lassen. Die Jugendlichen mussten sich im Stehen vorstellen und von ihren Straftaten berichten, wobei sie von den Gefangenen unterbrochen und korrigiert wurden. Sämtliche Behauptungen, sich ändern zu wollen, wurden als Lügen und »Blabla« zurückgewiesen. Manche Jugendliche erfuhren auch Demütigungen, wie Schuhe von Gefangenen polieren zu müssen. Eine Toilette ohne Abfluss wurde gezeigt, und der entwürdigende Charakter dortiger Drogenkontrollen beschrieben. Einzelne Jugendliche wurden gezwungen darauf zu sitzen, andere gegen ihren Willen auf einem Bett mit Gurten gefesselt. All dies diente dazu, die Hölle des Gefängnisses und die Notwendigkeit einer Umkehr zu veranschaulichen. Den weiblichen Besucherinnen des Männergefängnisses wurde gedroht, im Frauengefängnis sei es keineswegs besser und das Beispiel einer Frau er- zählt, die dort mehrfach mit einer Flasche vergewaltigt worden war.

Aus Interviews mit den Jugendlichen ließ sich ableiten, dass der Besuch in Ullersmo weitgehend als gleichzeitig aufregend und unangenehm sowie anfangs als beängstigend wahrgenommen wurde. Das galt allerdings keineswegs für alle, die daran teilgenommen hatten. Einige sahen die Konfrontation als ein Spiel, und durch die vorherigen Berichte anderer Bewohner der entsendenden Institution geriet sie zur Herausforderung und zur Mutprobe als Voraussetzung echter Gruppenzugehörigkeit. Manche beschrieben das Gefühl, dass das Gefängnis gar nicht so schlimm sei, wie von den Gefangenen dargestellt. Mehrere Jugendliche brachten zum Ausdruck, die Gefangenen als Helden oder Vorbilder zu sehen. Einige Mädchen schwärmten anschließend von einem Gefangenen, in einem Fall entwickelte sich sogar eine kurze sexuelle Beziehung nach dem Vorbild von Bonny und Clyde.

Gestärkt wurde mit dem Programm das Gemeinschaftsgefühl zwischen Gefangenen und Strafvollzugsbediensteten, und die Gefangenen entwickelten mehr Selbstvertrauen in sich selbst und ihre positiven Fähigkeiten. Ein Vertreter der entsendenden Institution meinte auch einen drastischen Rückgang registrierter Straftaten zu erkennen. Allerdings sahen die meisten Jugendlichen solche positiven Effekte nicht und glaubten auch nicht daran, dass das Projekt ihr Verhalten ändern würde - eine nicht nur angesichts der US-amerikanischen Studien vermutlich zutreffende Selbsteinschätzung.

Bereits vor Beginn der Evaluation wurde das Ullersmo-Projekt von Fachleuten aus der Kriminologie, Sozialwissenschaft und Psychologie einhellig kritisiert, und zwar nicht nur wegen der Zweifel an seiner kriminalpräventiven Wirksamkeit, sondern auch aufgrund ethischer Bedenken. Dementsprechend hatte auch die Evaluation eine breiter angelegte Fragestellung als in den USA: Selbst wenn sich ein kriminalpräventiver Effekt hätte nachweisen lassen, stellte sich noch die Frage, ob die eingesetzten Mittel diesen Zweck rechtfertigen könnten. Mit einem experimentellen Design entsprechend 
den US-Studien wäre diese Frage schwerlich zu beantworten gewesen. Die qualitative Studie hingegen eröffnete einen verstehenden Blick auf die im Rahmen des Programms stattfindenden Interaktionen. Die Veröffentlichung der Studie mit der Darstellung demütigender und ängstigender Elemente führte dann auch zu weiteren Protesten und zum Abbruch des Programms.

\section{Fortgesetzte Verbreitung der Programme und der erzieheri- sche »Twist« der Programme}

Mag mancher in den 80er- und frühen 90er-Jahren noch den Eindruck gehabt haben, dass die erdrückende Beweislast gegen die Programme deren Ende auch in den USA und anderen englischsprachigen Ländern bedeuten würde, so ist zumindest seit Ende der 90er-Jahre klar, dass dies nicht der Fall ist. Zwar wurden von der Vielzahl der "Scared Straight«Programme, die sich nach der Ausstrahlung der TV-Dokumentation auf über 30 Bundesstaaten der USA ausgedehnt hatten, auch einige eingestellt, andere aber wurden modifiziert oder schlicht beibehalten, und es kamen sogar ganz neue ebenso hinzu wie schließlich sogar gesetzliche Verpflichtungen, solche Gefängnisbesuche anzubieten (Finckenauer / Gavin 1999, S. 123 ff., Petrosino et al. 2003, S. 3). Über die Massenmedien werden nach wie vor Erfolgsmeldungen verbreitet.

Bei der Evaluation des australischen Programms "Stay Straight « aus Victoria zeigte sich, dass es auch andere als kriminalpräventive Intentionen sein können, die zur Bestandskraft der Programme beitragen. Dort waren negative Ergebnisse bezüglich der Abschreckungswirkung früherer australischer Gefängnisbesuchsprogramme bereits bekannt, als sich ein Gefangener mit der falschen Behauptung, eines davon habe eine 70\%ige Erfolgsrate aufgewiesen und mit dem Anliegen, ein solches Programm neu zu etablieren, an die Verantwortlichen wendete. Nachdem diese zunächst unter Hinweis auf frühere Misserfolge mit und Kontroversen über solche Programme ablehnten, gab der »Director General of Corrections « schließlich wider besseren Wissens nach, offenbar weil es dem Gefangenen gelungen war,
1.500 befürwortende Briefe von Personen des öffentlichen Lebens vorzulegen. Begründet wurde diese Kehrtwende mit der erwarteten Wirkung nicht des Besuchs selbst, sondern seiner Nachbereitung, deren halbherzige Umsetzung aber dafür spricht, dass es sich dabei nur um eine vorgeschobene Rechtfertigung handelte. Zudem beklagten sich später Jugendliche darüber, von Strafvollzugsbediensteten misshandelt worden zu sein, bei anderen gab es Hinweise darauf, dass sie durch den Besuch so erfolgreich verängstigt wurden, dass eine Traumatisierung zurückblieb. Geplant war die Programmteilnahme als Voraussetzung für Entlassung aus der Untersuchungshaft, praktisch wurde sie aber zu einer Netzerweiterung strafrechtlicher Sanktionen, indem sie, wohl auch gerade aufgrund ihrer Härte, von den Gerichten zunehmend als Strafmaßnahme angeordnet wurde. Die Anschuldigungen wegen Tätlichkeiten führten schließlich dazu, dass das Programm gestoppt werden konnte.

Ungeachtet der Evaluationen und der Kritik an den Programmen aus humanitärer Perspektive, bestehen diese fort, werden von ihren heutigen Protagonisten aber stets von den früheren Programmen und der Intention von "Scared Straight « abgegrenzt. Jugendliche zu verängstigen, wird als Ziel zurückgewiesen, stattdessen eine (nur freundlichere) erzieherische Absicht in Anspruch genommen. Es wird behauptet, lediglich die Einschüchterung habe sich als wirkungslos erwiesen, wohingegen eine weniger brutale Pädagogik bezogen auf den selben Inhalt - mit den bekannten Erfolgsmeldungen versehen wird. Diese Behauptungen werden völlig ungetrübt von der Tatsache weiterhin verbreitet, dass sie sämtlich bereits im Zusammenhang mit einer Mehrzahl der negativ evaluierten Programme erhoben worden waren. Das gilt gerade auch für die Behauptung, ein Programm sei weniger konfrontativ als »Scared Straight « und eher erzieherisch ausgerichtet. Da es jenseits dieser Behauptungen alle Programme auszeichnet, auf Erziehung durch Angst vor Strafe zu setzen, ist der vermeintlich »erzieherische Twist« der Programme auch theoretisch unplausibel und wird als bloßer Rechtferti- gungsmechanismus gegenüber Evaluation und Kritik erkennbar.

\section{Das »Panacea-Phänomen«}

Trotz entgegenstehender Evaluationsergebnisse, und obwohl die Programme aus der Wissenschaft in der Regel wenig Unterstützung und viel Kritik erfahren, geht von ihnen offensichtlich eine beständige Faszination aus. Diese versuchte Finckenauer bereits 1982 im Hinblick auf das damals und bis heute fortbestehende JAP mit dem »Panacea-Phänomen « zu erklären: Gerade bei besonders komplexen sozialen Problemen, wie dem der Jugenddelinquenz, bestünde ein besonders starkes gesellschaftliches Bedürfnis nach extrem einfachen Lösungen, nach einem umfassend einsetzbaren und zugleich billigen Allheilmittel. Daher würden im politischen Diskurs immer neue solcher Wundermittel mit unrealistischen Versprechungen angepriesen, auf die zwingend deren Enttäuschung folgen müsse, die dann in einem ewigen Zirkel zur Suche nach einem neuen Panacea führe (Finckenauer 1982, S. 3 ff.).

Als probates Gegenmittel empfahl Finckenauer (1982, S. 233) Evaluationen mit experimentellem Forschungsdesign. 17 Jahre später korrigierte sich Finckenauer unter dem Eindruck der Bestandskraft von Gefängniskonfrontationsprogrammen, die sich zwar regelmäßig von der Einschüchterungsstrategie des »Scared Straight « im Namen eher erzieherischer Aspekte distanzieren, diesem aber bei näherem Hinsehen dennoch verblüffend ähneln (Finckenauer/Gavin 1999, S. 13 ff.; 123 ff.) teilweise. Er hält es nunmehr nicht mehr für zwingend, dass ein Panacea nach Enttäuschung durch ein neues abgelöst wird, sondern auch für möglich, dass es dennoch fortbesteht. Es werde auch oft auf ähnliche, aber neue Programme zurückgegriffen, die auf denselben impliziten theoretischen Annahmen beruhten. Experimentelle Forschungsdesigns hält er allerdings nach wie vor für dagegen indiziert, obwohl diese in ihrer Theorielosigkeit oft den Gefängnisbesuchsprogrammen gleichkommen, selbst ethisch bedenklich sind (Graebsch 2000) und keine Hinweise erkennbar sind, dass ihre Ergebnisse stärkere Berücksichtigung finden als die anderer Studien (Graebsch 2004).

Die Gefängnisbesuchsprogramme in Deutschland geben vor, sich von den US-amerikanischen grundlegend zu unterscheiden. Dafür werden aber die international bekannten und hier wie dort nicht tragfähigen Unterschiede zwischen Abschreckung und Erziehung (durch Abschreckung) herangezogen. Oder es wird zur vermeintlichen Abgrenzung sogar so völlig Unrichtiges behauptet, wie dass die Gefangenen in den US-Projekten kein Interesse an Gesprächen mit den Jugendlichen gehabt hätten, nicht $\mathrm{zu}$ ihren Taten stehen konnten bzw. überhaupt keine Begegnung mit Gefangenen stattgefunden hätte. Negative Evaluationsergebnisse werden als für das eigene Projekt ungültig abgewehrt, offensichtlich ebenfalls ohne sie auch nur zu kennen (vgl. zu alledem Böhm 2003, S. 17; Böhm / Ruhe 2003). Demgegenüber spricht nichts dafür, dass die Programme in der Bundesrepublik eine andere Wirkung entfalten könnten als anderswo auf der Welt. Wenn auch gehofft werden kann, dass traumatisierende Einwirkungen auf die Jugendlichen hierzulande bislang nicht vorgekommen sind, so stellt doch das gesamte angstauslösende Setting, wie es durch die Programme geschaffen wird, eine Situation her, in der Grenzverletzungen und Übergriffe nahe liegen. Wo es sich bewahrheitet, dass die Konfrontation eher ein freundliches Gespräch denn ein Schockerlebnis darstellt, ändert dies nichts an der zugrundeliegenden Annahme, es sei das mangelnde Wissen über die Ausgestaltung und Härte einer Gefängnisstrafe, die die Jugendlichen (weiterhin) mit Straftaten in Erscheinung treten lasse. Die dabei vielfach genannten Ziele »Erziehung « und »Information « genügen für eine Abgrenzung von "Scared Straight" nicht, denn beide lassen sich nicht ohne einen Inhalt denken, der dabei vermittelt werden soll. Werden aber Informationen über die einschneidende Wirkung einer Gefängnisstrafe an Jugendliche gegeben, die nach dem Kriterium ihrer eigenen Delinquenzgefährdung für die Teilnahme ausgesucht wurden, ist schwer vorstellbar, was Intention und theoretische Fundierung sein sollten, wenn nicht die, zu Abschreckungszwecken mit Informationen 
über mögliche Konsequenzen auf späteres Verhalten der TeilnehmerInnen einzuwirken. Vielleicht spricht sogar einiges dafür, dass die Programme in dem Maße, in dem sie sich von einer echten Konfrontation hin zu einer informierend-pädagogischen Ausrichtung orientieren, wie sie auch etwa das Filmprojekt »Aufschluss « des Maßstab e.V. in ganz ähnlicher Weise verfolgt (vgl. dazu Kawamura-Reindl 2004), den Vorteil einbüßen, mit dem sie doch einst angetreten waren: Dem bereits als wirkungslos hinlänglich bekannten pädagogischen Zeigefinger ein Erlebnis entgegenzusetzen, das die Jugendlichen in ihrer eigenen Lebenswelt erreicht. Es spricht demgegenüber nichts dafür, dass durch die Orientierung an freundlicher Pädagogik plötzlich doch ein Abschreckungseffekt an die Stelle des Vorbildeffekts tritt. Das von den Programmen schließlich auch irgendwie intendierte Lernen der Jugendlichen am Modell der Gefangenen lässt sich ganz offensichtlich, wo es funktioniert, nicht auf den letzten Schritt in deren Lebensgeschichte, der als Umkehr präsentiert wird, beschränken. Die Jugendlichen nehmen sich dann wohl lieber gleich die gesamte Lebensgeschichte zum Vorbild.

\section{Aversionsprogramme im Kon- text kriminalpolitischer Ent- wicklungen}

Die Gefängnisbesuchsprogramme, so erfolglos oder auch kontraproduktiv sie sich in kriminalpräventiver Hinsicht darstellen, bieten ein höchst erfolgreiches Konzept an, wie über Kriminalität in Politik und Medien in einer Zeit geredet werden kann, in der sich ein Wandel weg vom Resozialisierungsgedanken hin zu Abschreckung und Schuldausgleichsgedanken vollzieht (dazu auch schon Cavender 1981). Ein Schlüssel zum Verständnis, weshalb die Gefängnisbesuchsprogramme überdauern, liegt dabei gerade in der Härte der Programme und vor allem auch des in ihnen präsentierten, realen Gefängnislebens, die doch eigentlich schon aus ethischer Sicht und unabhängig von den Evaluationsergebnissen Kritik hervorrufen müsste. Die Programme sind für eine Kriminalpolitik höchst funktional, die Verantwortung für soziale Probleme beim kriminellen Individuum und dessen Fehlentscheidung verortet und gnadenlosen, zeitlich unbegrenzten Strafvollzug als quasi-natürliche Konsequenz favorisiert. Denn diese Botschaft wird in den Gefängniskonfrontationsprogrammen öffentlichkeitswirksam sogar von den Gefangenen selbst verbreitet. Wenn aber diese schon die Schuld alleine bei sich selbst suchen, wenn schon diese Gefangenen selbst die Härte der Haftbedingungen bis hin zu alltäglicher sexualisierter Gewalt nicht für kritikwürdig halten, sondern für die normale Folge eines verpfuschten Lebens, gegen das man sich nur im jugendlichen Alter noch hätte entscheiden können, wer sonst sollte dann dagegen noch etwas einwenden oder gegen die Haftbedingungen protestieren? Sogar gesetzeswidrige Eskalationen des Strafvollzugs werden damit zu dessen Rechtfertigung verwendetund zwar durch die Gefangenen selbst.

Einiges spricht dafür, dass die Programme positive Effekte immerhin für die beteiligten Gefangenen haben können, u. a. für deren Selbstvertrauen (vgl. dazu auch Keller 1993). Als Vorteil kann es diesbezüglich schon betrachtet werden, wenn sich die involvierten Gefangenen gerne und freiwillig beteiligen. Selbstverständlich aber dürfen solche Vorteile nicht mit zweifelhaften Wirkungen für die Jugendlichen erkauft werden. Der Fokus auf solche kriminalpräventiv intendierte Gefängnisbesuchsprogramme ist demgegenüber Teil einer allgemeinen unsäglichen Entwicklung weg von der Sozial- und hin zur Kriminalpolitik, wenn sinnbringende Resozialisierungsprogramme für Gefangene nur ermöglicht werden, sofern sie einerseits keine Kosten und andererseits noch einen kriminalpräventiven Effekt versprechen. Als Alternative für die Gefangenen kommen Programme in Betracht, in denen diese ganz allgemein die Öffentlichkeit, etwa Studierende der Rechtswissenschaft oder auch SchülerInnen über das Leben im Strafvollzug und Wege dorthin informieren. Solche Veranstaltungen müssten dann allerdings für ganz unterschiedliche Gefangene offen sein, nicht nur die sich als geläutert präsentierenden, wie es sich bei den kriminalpräventiv orientierten Programmen aus deren Ansatz ergibt.
Die Verfasserin arbeitet am Zentrum für feministische Studien der UniversitätBremen zfs@uni-bremen.de.

\section{Literatur}

Böhm, Christian (2003): »Schulische Gewaltprävention. Konfrontation, Vermittlung und Kooperation.«, in: Kerner, H.-J./Marks, E.: Internetdokumentation Deutscher Präventionstag Hannover, verfügbar unter http://www.praeventionstag.de

Böhm, Christian / Ruhe, Volkert (2003): "Aktueller Stand des GHJ Projekts.", verfügbar unter: http://www.gefangenehelfen-jugendlichen.de/sides/ aktuell.php vom 1.9.2003.

Campbell Collaboration Research Review Policy Brief, 28. November 2003, verfügbar unter

http://www.campbellcollaboration.org /doc-pdf/ssrpm.pdf

Cavender, Gray (1981): »Scared Straight $«$ : Ideology and the Media. «, in: Journal of Criminal Justice 9, S. 431-439.

Cook, David Dykes / Spirrison, Charles L. (1992): "Effects of a Prisoner-operated delinquency Deterrence Program: Mississippi's Project Aware. «, in: Journal of Offender Rehabilitation Vol. 17, 3/4, S. 89-99.

Finckenauer, James O. (1982): „Scared Straight! and the Panacea Phenomenon.«, Englewood Cliffs, New Jersey: Prentice-Hall.

Finckenauer, James O. / Gavin, Patricia W. (1999) with Hovland, Arild / Storvoll, Elisabet: »Scared Straight. The Panacea Phenomenon Revisited.«, Prospect Heights, Illinois: Waveland Press.

Graebsch, Christine (2000): »Legal Issues of Randomized Experiments on Sanctioning. «, in: Crime \& Delinquency, Vol. 46, No. 2, April, S. 271-281.

Graebsch, Christine (2004): »,Evidence-Based Crime Prevention': Anspruch und Praxisbeispiele einer Kriminalpolitik nach medizinischem Modell.«, in: Kriminologisches Journal 36. Jg., Heft 4, S. 266-283.

Homant, Robert J./Osowski, Gregory (1981): "Evaluation of the "Scared Straight Model: Some Methodological and Political Considerations. «, in: Corrective and Social Psychiatry and Journal of Behavior Technology Vol. 27, S. $130-134$.

Kawamura-Reindl, Gabriele (2004): »Aufschluss « und »Knast - Ich? - Nie!«. Ein Filmprojekt und eine Broschüre zum Film.", in: Neue Kriminalpolitik, Jg. 16, Heft 2, S. 49.

Keller, Robert L. (1993): »Some Unanticipated Positive Effects of a Juvenile Awareness Program on Adult Inmate Counselors. «, in: International Journal of Offender Therapy and Comparative Criminology 37(1), 75-83.

Lewis, Roy V. (1981): »The Squires of San Quentin: an Evaluation of a Juvenile Awareness Program.«, Department of the Youth Authority, State of California.
Lewis, Roy V. (1983): »Scared Straight - California Style: Evaluation of the San Quentin Squires Program. «, in: Criminal Justice and Behavior 10 (2), S. 209-226.

Lipsey, Mark W. (1992): »Juvenile Delinquency Treatment: A Meta-Analytic Inquiry into the Variability of Effects.", in: Cook, Thomas et. al., Hrsg.: »MetaAnalysis for Explanation: A Casebook.", New York: Russell Sage Foundation.

Locke, Thomas P./Johnson, Glenn M./ Kirgin-Ramp, Kathryn/Atwater, Jay D. / Gerrard, Meg (1986): »An Evaluation of a Juvenile Education Program in a State Penitentiary.«, in: Evaluation Review Vol.10, no.3, June, S.281-298.

Lundman,Richard J. (2001; Vorauflage 1993): »Prevention and Control of Juvenile Delinquency.«, 3. Auflage, New York u. a.: Oxford University Press.

Muhammad, Larry (1999): »Kids and crooks revisited: Some were ,Scared Straight!' «, in: USA Today, April 12, S. 4 D.

Petrosino, Anthony / Turpin-Petrosino, Carolyn / Buehler, John (2003): »,Scared Straight' and other Juvenile Awareness Programs for Preventing Juvenile Delinquency«, Updated C2 Review, in: The Campbell Collaboration Reviews of Intervention and Policy Evaluations (C2Ripe), November, Philadelphia, Pennsylvania: Campbell Collaboration, verfügbar unter:

http://www.campbellcollaboration.org/ doc-pdf/ssrupdt.pdf

Petrosino, Anthony / Turpin-Petrosino, Carolyn / Finckenauer, James O. (2000): »Well-Meaning Programs Can Have Harmful Effects! Lessons From Experiments of Programs Such as Scared Straight.«, in: Crime\&Delinquency Vol. 46, No. 3, S. $354-379$.

Sherman, Lawrence W. / Farrington, David P./Welsh, Brandon C./MacKenzie, Doris Layton (2002), Hrsg.: »EvidenceBased Crime Prevention«, London u.a.: Routledge.

Sherman, Lawrence W. / Gottfredson, Denise C. / MacKenzie, Doris L./Eck, John / Reuter, Peter / Bushway, Shawn D. (1997): Preventing Crime: What Works, What Doesn't, What's Promising. A Report to the United States Congress. Prepared for the National Institute of Justice.«, verfügbar unter http://www.preventingcrime.org

\section{Anmerkungen}

1 Man kann nun berechtigte Kritik an der offiziellen Registrierung von Straftaten als Maß für Kriminalität und kriminalpräventiven Erfolg üben. Es handelt sich dabei aber um einen durchaus zuverlässigen Indikator für die Entwicklung der Größe, die zu beeinflussen von den Befürwortern des Programms behauptet wird. Sie eignet sich daher trotz aller Probleme zur empirischen Überprüfung kriminalpräventiver Wirkungsbehauptungen. 